\title{
Evaluation of Compact Real-time Descriptors for Camera Motion Estimation
}

\author{
Shota Ogawa, Kenichi Asami, and Mochimitsu Komori \\ Department of Applied Science for Integrated System Engineering, Kyushu Institute of Technology \\ 1-1 Sensui-cho, Tobata-ku, Kitakyushu, Fukuoka 804-8550, Japan \\ E-mail: ogawa@es.ise.kyutech.ac.jp
}

\begin{abstract}
This paper introduces the image processing system of camera motion estimation for autonomous mobile robots. Most autonomous mobile robots need to quickly recognize the surroundings so as to move to the space. Moreover, the image processing algorithm requires low computation complexity. Accordingly, we evaluate an image processing system based on Compact and Real-time Descriptors $(\mathrm{CARD})^{(1)}$. Proposed system has scale-invariance and rotation-invariance for local image feature. Our method uses a multi-scale image pyramid to extract coordinates for keypoint and a log-polar binning pattern for a patch around the keypoint. The results of correspondence for keypoint between two images were good enough to estimate the camera motion.
\end{abstract}

Keywords: scale- and rotation-invariance, gradient features, feature description, log-polar binning pattern.

\section{Introduction}

Local feature descriptor is effective for visual correspondence between two images. Scale-Invariant Feature Transform $(\text { SIFT) })^{(2)}$ is representative method for local feature descriptors and has been verified to be useful for image recognition application. But it is unsuitable to realtime processing for autonomous mobile robots. It is important for robot application to reduce computational cost for the mobile ability.

This paper focuses on scale- and rotation-invariant feature descriptor by using a multi-scale image pyramid and a log-polar binning pattern, instead of a big amount of computation of gradient extremum search and a binning pattern manipulation of SIFT descriptor.

\section{CARD Algorithm}

\subsection{Keypoint Detection}

Keypoint detection is an important function for scaleinvariance. SIFT is scale-invariant by using Difference of Gaussians (DoG) pyramid. But a DoG pyramid requires heavy processing because an image needs to be applied different sizes of Gaussian filter.

Instead of DoG pyramid, the method uses a multi-scale image pyramid to detect keypoint which is robust under scale change. That image pyramid is structured in the fast way of downsizing image. The size of the $n$-th level corresponds to the $1 / \sqrt{2}$ size of the $(n-1)$-th level. The 0 -th level represents original image. In practice, $n$-th level is represented by making half size of (n-2)-th level. Then Good Features to Track (GFtT) ${ }^{(3)}$ method detects the coordinates for keypoint from each level of the scale pyramid. GFtT is based on Harris corner detector.

\subsection{Description with Gradient Features}

The descriptor uses the gradient of corner points with the scale- and rotation-invariant distinctiveness. The gradient represents magnitude and direction for local pixel value. It is found from the difference of brightness value for neighborhood around the sampling point. Also, this method is strong in a local illumination change. The set of gradients in a regional block characterizes the local feature description.

The descriptor use a log-polar binning pattern which shows the patch around a keypoint for feature description, in the same way of $\mathrm{GLOH}^{(4)}$. A patch around a keypoint is usually relocated before feature description when the image is rotated. The employment of a log-polar pattern for the patch around a keypoint avoids many bilinear interpolations. 

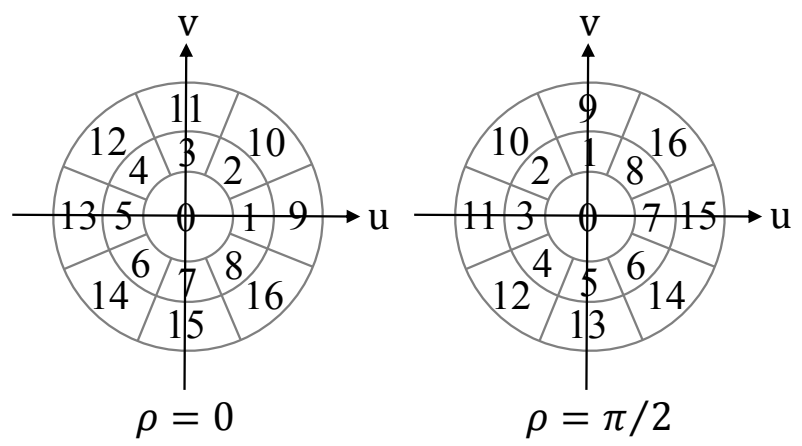

Fig. 1. A log-polar binning pattern.

The center of a log-polar binning pattern indicates a keypoint and the binning pattern divides into 17 blocks around it. The descriptor enables to rotate this pattern along to the gradient of keypoint (principal orientation) for rotation-invariance. Thus a parametric function $\psi(u, v, \rho)$ to represent rotated binning pattern is introduced, where $\rho$ denotes the principal orientation in radian and $(u, v)^{T}$ denotes a relative coordinate from the keypoint. The descriptor rotates the binning pattern on $u v$-plane by $\rho$. The function $\psi(u, v, \rho)$ returns the number of the block. A binning pattern example of $\psi(u, v, \rho)$ is shown in Fig. 1. The block number $k$ for a pixel $(x, y)^{T}$ can be determined as follows:

$$
k=\psi\left(x-p_{x}, y-p_{y}, \rho\right),
$$

$\left(p_{x}, p_{y}\right)^{T}$ denotes an image coordinate of a keypoint.

An orientation of the pixel $(x, y)^{T}$ is quantized to $N$ levels to obtain the gradient histograms of a keypoint. An angle quantization function is given as follows:

$$
Q_{N}(\theta)=\left\lfloor\frac{N \theta}{2 \pi}+\frac{1}{2}\right\rfloor \bmod N
$$

$\theta$ is a radian angle in the range $[0,2 \pi)$ and which quantizes an angle in radian to an integer from 0 to $N-1$. The value of $N$ is set to 8 in this paper.

When the binning pattern is rotated, all the gradient of the pixel must be shifted around into $\rho$. This calculation can be represented as follows:

$$
l=Q_{N}(\theta(x, y)-\rho),
$$

where $l$ determines the quantized gradient direction in the gradient histogram of a keypoint and $\theta(x, y)$ returns the angle to a vector $(x, y)^{T}$ in the range $[0,2 \pi)$. Here the angle has the origin $\left(p_{x}, p_{y}\right)^{T}$ and takes the direction of counter clockwise from positive $\mathrm{x}$-axis. In this paper, the quantized grades for binning pattern and gradient direction are unified. The parametric representation of a log-polar binning pattern is defined as follows:

$$
\begin{aligned}
& \psi(u, v, \rho)= \\
& \left\{\begin{array}{cc}
0 & , 0 \leq r<r_{1} \\
1+Q_{8}(\theta(u, v)-\rho) & , r_{1} \leq r<r_{2}, \\
9+Q_{8}(\theta(u, v)-\rho) & , r_{2} \leq r \leq r_{3}
\end{array}\right.
\end{aligned}
$$

where $r$ denotes the distance between $(u, v)^{T}$ and $\left(p_{x}, p_{y}\right)^{T}$. The thresholds $r_{1}, r_{2}$, and $r_{3}$ are set to the radial distances.

The feature descriptor $d$ is obtained as below.

1. Calculate the block number $k$ from (1) and the gradient direction $l$ from (3) for all pixels in a block.

2. Accumulate the gradient histogram $h_{k, l} \leftarrow h_{k, l}+$ $m(u, v) G_{\sigma}(u, v)$

3. Normalize $d$ according to the block area. where $m(u, v)=\sqrt{u^{2}+v^{2}}$ denotes the magnitude of the gradient and $G_{\sigma}$ denotes the weighted values as a normal distribution with variance $\sigma$. In the case of 17 blocks and 8 quantized directions, the 136 dimensional descriptor is constructed.

In practice, computation of (1) can be done beforehand because $u, v$, and $\rho$ take only a finite number. Therefore, the lookup table for $\psi(u, v, \rho)$ is prepared to reduce the computational cost.

\section{Matching Algorithm}

\subsection{Rotation of a Binning Pattern}

As mentioned in the preceding section, the descriptor rotate a binning pattern for rotation invariance. For that purpose, a principal orientation is calculated from the whole gradient histogram by extracting $d$ for all the pixels in 17 blocks. Consequently, the whole gradient histogram can be easily accumulated the values of gradient magnitude. The principal orientation is assigned to the gradient direction with the maximum gradient magnitude as shown in Fig. 2.

whole gradient histogram

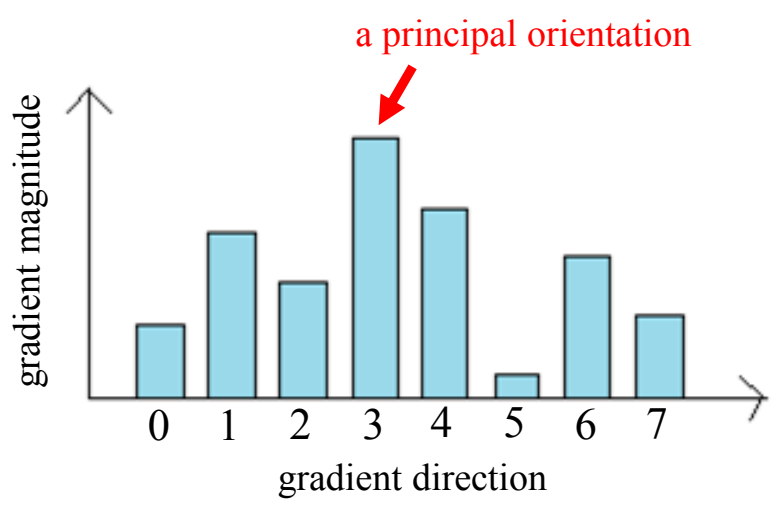

Fig. 2. Calculation of a principal orientation 


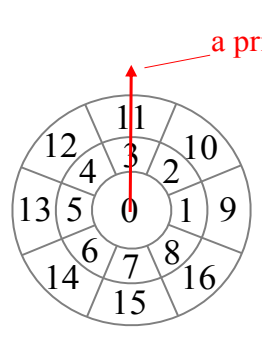

in reference image principal orientation

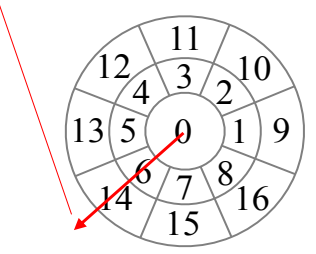

in alternative image

$\operatorname{turn} 135^{\circ}$

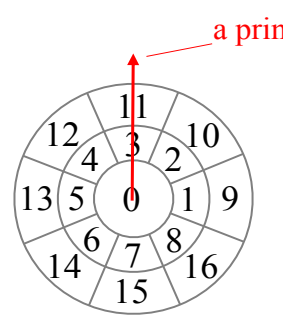

in reference image

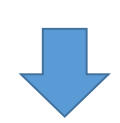

(2)

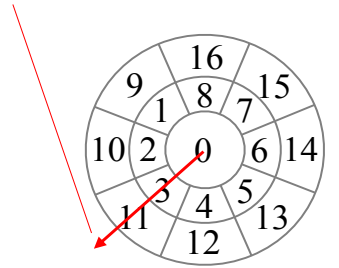

in alternative image
Fig. 3. Rotation of a log-polar binning pattern

In the matching, there are two images which are called reference image and alternative image respectively. A binning pattern of the alternative image is rotated to adjust the principal orientation of it to the reference image. At the same time, the orientation of all pixels in the binning pattern is rotated as shown in Fig. 3.

\subsection{Matching by Euclidean Distance}

The similarity among feature descriptions is measured by the ratio of the Euclidean distances to the two nearest neighbors so as to make robust matches. Accordingly, most mismatches are rejected by the matching result.

For every image of a multi-scale pyramid, the feature descriptions is evaluated for matching keypoints. Therefore, the scale change by a camera motion can be handled.

\section{Experiments}

Several experiments measured the correct matching rate and the error matching rate for changing the location and turning camera. Before showing the result of experiments, the correct matching rate and the error matching rate are defined. They are represented as follows:

correct matching rate $=$

$$
\frac{\text { number of true correspondances }}{\text { number of keypoints }} \times 100
$$

error matching rate $=$

$$
\frac{\text { number of false correspondances }}{\text { number of keypoints }} \times 100
$$

where the number of keypoints is counted in the reference image at the 0 -th level. The number of true correspondences represents the true positive of the matching result. The number of false correspondences represents the false positive.

\subsection{Location Change}

The camera position was changed to right, left, forward, and backward perspectives. The matching correspondences in the images are shown in Fig. 4. The results of matching rate are shown in Table 1.

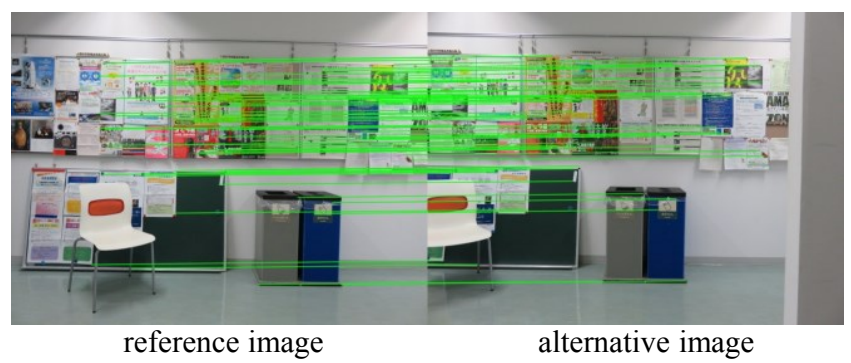

(a). Right perspective change

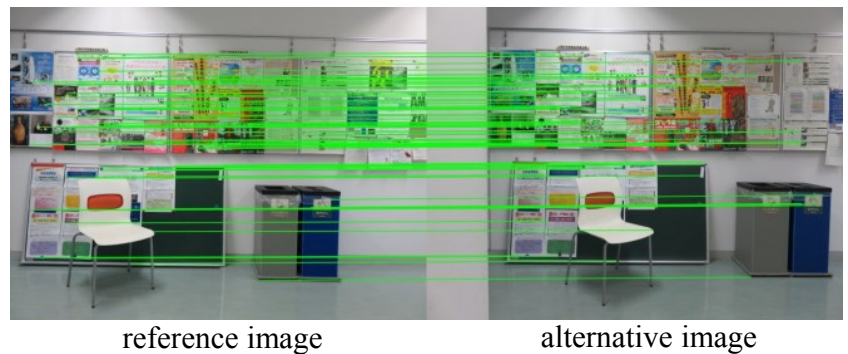

(b). Left perspective change

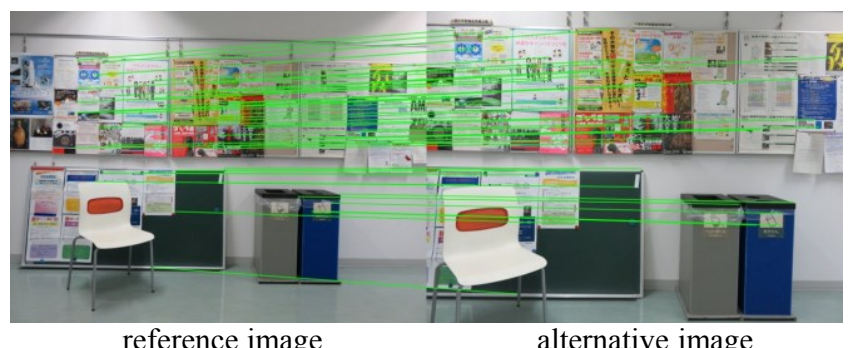

(c). Forward perspective change

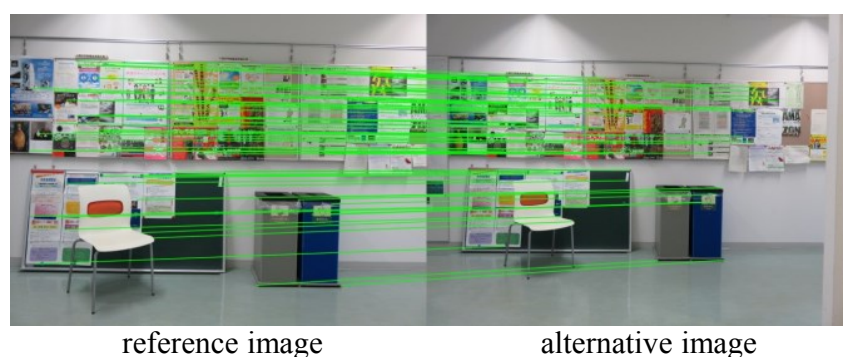

(d). Backward perspective change

Fig. 4. Matching result in location change 
Table 1. Result for location change.

\begin{tabular}{|c|c|c|}
\hline & $\begin{array}{c}\text { Correct } \\
\text { matching rate[\%] }\end{array}$ & $\begin{array}{c}\text { Error } \\
\text { matching rate[\%] }\end{array}$ \\
\hline $\begin{array}{c}\text { Right } \\
\text { perspective change }\end{array}$ & 82.0 & 0.0 \\
\hline $\begin{array}{c}\text { Right } \\
\text { perspective change }\end{array}$ & 86.5 & 0.0 \\
\hline $\begin{array}{c}\text { Right } \\
\text { perspective change }\end{array}$ & 55.0 & 2.0 \\
\hline $\begin{array}{c}\text { Right } \\
\text { perspective change }\end{array}$ & 78.7 & 1.4 \\
\hline
\end{tabular}

The correct matching rate achieved $82.0 \%$ and error matching rate showed $0.0 \%$ for right movement. The correct matching rate achieved $55.0 \%$ and the error matching rate showed $1.1 \%$ for forward movement. Accordingly, this algorithm verified robust for scale and view change.

\subsection{Angle Change}

The camera angle was changed to clockwise incrementally. The matching correspondences are shown in Fig. 5. The results of matching rate are shown in Table 2.

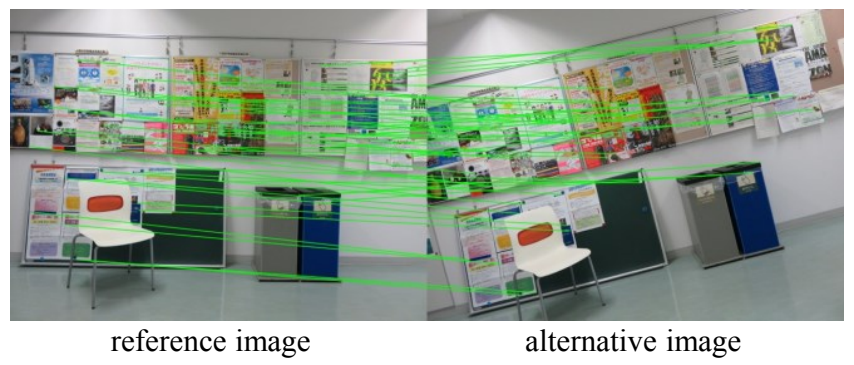

(a). 15 degree angle change

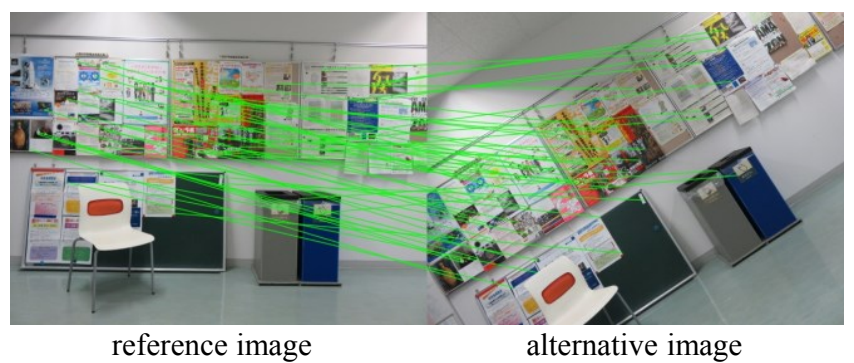

(b). 30 degree angle change

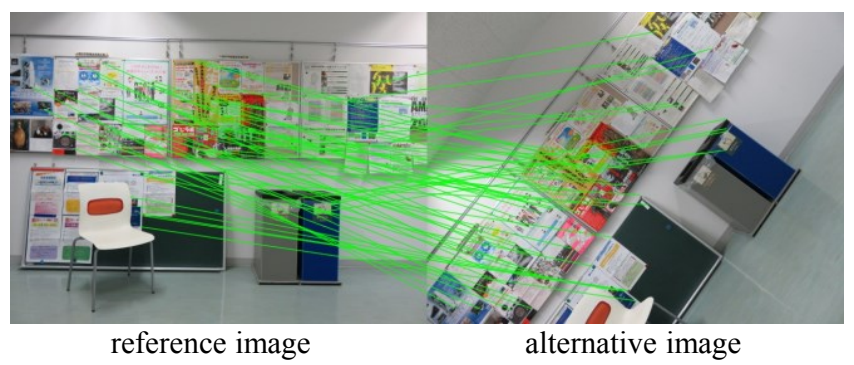

(c). 45 degree angle change

Fig. 5. Matching result in angle change
Table 2. Result for angle change

\begin{tabular}{|c|c|c|}
\hline & $\begin{array}{c}\text { Correct } \\
\text { matching rate[\%] }\end{array}$ & $\begin{array}{c}\text { Error } \\
\text { matching rate[\%] }\end{array}$ \\
\hline $\begin{array}{c}\text { 15 degree } \\
\text { angle change }\end{array}$ & 56.2 & 0.0 \\
\hline $\begin{array}{c}30 \text { degree } \\
\text { angle change }\end{array}$ & 58.4 & 0.0 \\
\hline $\begin{array}{c}45 \text { degree } \\
\text { angle change }\end{array}$ & 70.8 & 0.0 \\
\hline
\end{tabular}

The correct matching rate achieved $56.2 \%$ and the error matching rate showed $0.0 \%$ for angle change of 15 degree. The correct matching rate increased $70.8 \%$ for angle change of 45 degree. That's because a binning pattern is divided by 45 degree. As the result, this algorithm verified robust for rotation.

\section{Conclusion}

In this paper, we evaluated an image processing system based on CARD. For three different scenes, experiments including Fig. 4 and Fig. 5 were operated to confirm the robustness for the camera motion changes. The experiments for location and angle changes showed that the correct matching rate was high and the error matching rate limited to $1.1 \%$. This rates are sufficient for autonomous mobile robots. The processing time of this algorithm should be reduced. We would like to propose the binarized algorithm of this descriptor based on FREAK ${ }^{(5)}$.

\section{References}

(1) Mitsuru Ambai, and Yuichi Yoshida : "CARD: Compact And Real-time Descriptors", International Conference on Computer Vision, pp. 97-104, 2011.

(2) Vanderlei Bonato, Eduardo Marques, and George A. Constantinides : “A parallel Hardware Architecture for Scale and Rotation Invariant Feature Detection", IEEE Transactions on Circuits and System for Video Technology, Vol. 18, No. 12, pp. 1703-1712, 2008.

(3) Jianbo Shi, and Carlo Tomasi : "Good Features to Track", IEEE Conference on Computer Vision and Pattern Recognition, pp. 592-600, 1994.

(4) Krystian Mikolajczyk, and Cordelia Schmid : "A Performance Evaluation of Local Descriptors", IEEE Transactions on Pattern Analysis and Machine Intelligence, Vol. 27, No. 10, pp. 1615-1630, 2005.

(5) Alexandre Alahi, Raphael Ortiz, and Pierre Vandergheynst : "FREAK: Fast Retina Keypoint”, IEEE Conference on Computer Vision and Pattern Recognition, pp. 510-517, 2012. 